\title{
STAR AND GLOBULAR CLUSTER FORMATION
}

\section{IN MERGERS}

\author{
UTA FRITZE - V. ALVENSLEBEN \\ Universitäts-Sternwarte Göttingen \\ Geismarlandstr.11, 37083 Göttingen, Germany \\ ufritze@uni-sw.gwdg.de
}

The high burst strengths and star formation efficiencies found with spectrophotmetric and chemical evolutionary synthesis for mergers of gas-rich spirals led us to expect the formation of a secondary population of globular clusters (GC) with enhanced metallicity (F. - v. A. \& Gerhard 1994, A\&A 285, 751 u.775). HST imaging of NGC 7252, NGC 4038/39 and NGC 1275 revealed rich populations of bright young star clusters (YSC).

We model the evolution of colours, luminosities, stellar absorption features, and spectra for star clusters of various initial metallicities and find that metallicity effects are of particular importance in early phases. Comparison with spectroscopy of the brightest GC candidates in NGC 7252 confirms their high metallicity $Z \sim 0.01$ (F. - v. A. \& Burkert 1995, A\&A $300,58)$. Once metallicity is known, precise age dating becomes possible. With ages of YSCs precisely known, our models follow their luminosity and colour evolution over a Hubble time. We derive mean ages for the YSCs of $1.37_{-0.8}^{+1.6} \mathrm{Gyr}$ in NGC 7252 , in agreement with the global starburst age, and of $\gtrsim 0.2 \mathrm{Gyr}$ in Antennae. With their present age and a mean effective radius $\left\langle R_{\text {eff }}\right\rangle \sim 10 \mathrm{pc}$ (upper limit $\longrightarrow$ Meurer 1995, Nat 375, 742), the YSCs in NGC 7252 can indeed be expected to be GCs. They will fade over a Hubble time to about the luminosity of $\omega$ Cen. In Antennae, the LF of those YSCs with $R_{\text {eff }} \leq 10 \mathrm{pc}$, when evolved over a Hubble time, is found to look similar to the Galactic GCLF, except for an overpopulation of the faintest bins, at variance with van den Bergh's (1995, ApJ 450, 27) results for the LF of all YSCs. Dynamical effects - strongest during the first Gyr and at the lowest mass (luminosity) bins - are expected to further change this LF and remain to be estimated (F. - v. A. \& Burkert 1995, in prep.). Acknowledgement This work is supported by the Deutsche Forschungsgemeinschaft, grant Fr 916/2-1. 